\title{
Synthesis of Zeolite Nanomolecular Sieves of Different Si/Al Ratios
}

\author{
Pankaj Sharma, Moon Hee Han, and Churl-Hee Cho \\ Graduate School of Energy Science and Technology, Chungnam National University, 99 Daehak-ro, Yuseong-gu, \\ Daejeon 305-764, Republic of Korea \\ Correspondence should be addressed to Churl-Hee Cho; choch@cnu.ac.kr
}

Received 28 September 2015; Revised 26 October 2015; Accepted 28 October 2015

Academic Editor: William Yu

Copyright (C) 2015 Pankaj Sharma et al. This is an open access article distributed under the Creative Commons Attribution License, which permits unrestricted use, distribution, and reproduction in any medium, provided the original work is properly cited.

\begin{abstract}
Nanosized zeolite molecular sieves of different $\mathrm{Si} / \mathrm{Al}$ ratios have been prepared using microwave hydrothermal reactor (MHR) for their greater application in separation and catalytic science. The as-synthesized molecular sieves belong to four different type zeolite families: MFI (infinite and high silica), FAU (moderate silica), LTA (low silica and high alumina), and AFI (alumina rich and silicafree). The phase purity of molecular sieves has been assessed by X-ray diffraction (XRD) analysis and morphological evaluation done by electron microscopy. Broad XRD peaks reveal that each zeolite molecular sieve sample is composed of nanocrystallites. Scanning electron microscopic images feature the notion that the incorporation of aluminum to MFI zeolite synthesis results in morphological change. The crystals of pure silica MFI zeolite (silicalite-1) have hexagon lump/disk-like shape, whereas MFI zeolite particles with Si/Al molar ratios 250 and 100 have distorted hexagonal lump/disk and pseudo spherical shapes, respectively. Furthermore, phase pure zeolite nanocrystals of octahedron (FAU), cubic (LTA), and rod (AFI) shape have been synthesized. The average sizes of MFI, FAU, LTA, and AFI zeolite crystals are 250, 150, 50, and $3000 \mathrm{~nm}$, respectively. Although the length of AFI zeolite rods is in micron scale, the thickness and width are of a few nanometers.
\end{abstract}

\section{Introduction}

Microporous aluminosilicate molecular sieves known as zeolites have regular arrays of uniformly sized pores, defined channel system, control density of active sites, large surface area, and ion-exchange property and admit molecules below a certain critical size into their internal space which makes them useful for many shape and size selectivity based applications [1,2]. Ion-exchange and catalytic and adsorptive properties of molecular sieve materials originate from acid sites which further arise from the presence of accessible hydroxyl groups associated with tetrahedral framework of aluminum in a silica matrix. Furthermore, Si/Al ratio of the molecular sieve affects its hydrophobicity/hydrophilicity which in turns affects its solvent dehydration and recovery performance [2-7]. The LTA membranes have already been commercialized for their greater application in alcohol dehydration because of their hydrophilic nature and high alumina content [8]. Furthermore, extensive studies have been made on MFI (silicalite-1, ZSM-5), FAU (zeolite Y), and
AFI $\left(\mathrm{AlPO}_{4}-5\right)$ zeolite materials for gas separation, catalytic reactors, petrochemicals, pervaporation, and even medical diagnostics applications [9-13]. Sometimes, zeolites suffer from low diffusion efficiency due to the small pore size, especially when they are used for applications associated with large molecules/separation process. Therefore, the efficiency of these processes can be significantly improved by switching to nanosized materials because the diffusion limitation can be greatly eliminated by using nanosized zeolite particles that would offer a shorter diffusion path as well as more active sites on the external surface [14]. Studies using nanosized zeolite crystals for esterification of cyclohexanol with acetic acid showed that the catalytic activity depends directly on the external surface area and improved porosity present in nanocrystalline ZSM-5 zeolite [15]. Particle size reduction to nanometer actually increases the ratio of external to internal atoms; therefore, zeolite nanoparticles possess large external surface area and higher activity [16]. Furthermore, pervaporation process has gained widespread acceptance in the chemical industry as an effective process for separation 
of azeotropic mixtures; however, the crystallinity of zeolites that provides these attractive features imposes challenges on film fabrication, for example, to minimize the effect of grain boundary defects on permeation properties and to avoid stress-induced crack formation [17]. These problems can only be solved by having nanosized zeolite crystals for suspension preparation and seed coating.

The numbers of nanomaterial synthesis strategies have been reported for liquid phase hydrothermal synthesis process such as biomimetic, microemulsion, microfluidic, microwave, sonochemical, and template techniques. All these techniques are well known. The microwave chemistry is a well-established technique in organic synthesis but its use in inorganic nanomaterials' synthesis is still far away from reaching its full potential. However, its rapidly growing use suggests that microwave chemistry will play an outstanding role in the broad field of nanoscience and nanotechnology [18]. Microwaves assisted syntheses of zeolite molecular sieves have many advantages such as high phase purity, fast crystallization, homogenous heating, and narrow particle size distribution. Furthermore, microwave hydrothermal synthesis process is not only helpful in the production of nanosized particles in shorter period of time but also useful in morphology controlled molecular sieves synthesis $[19,20]$.

As our research interest is mainly focused on the synthesis of high performance membrane (zeolite membrane, hybrid membranes, and superflux membranes) and novel gas adsorption materials, therefore, an attempt was made to synthesize nanosized microporous molecular sieves using microwave hydrothermal reactor by liquid phase synthesis route consisting of three steps. The selection of zeolite molecular sieves for synthesis was made on the basis of their widespread applicability in different fields of science. Moreover, selected molecular sieves have different $\mathrm{Si} / \mathrm{Al}$ ratio, pore structure, pore size, channel type, and morphologies.

\section{Experimental}

2.1. Materials and Phase Pure Nanomolecular Sieves Synthesis. The silica sources used for the preparation of hydrothermal reactant mixtures for zeolite molecular sieves synthesis were tetramethylammonium silicate (TMAS) $\left(\left(\mathrm{CH}_{3}\right)_{4} \mathrm{~N}(\mathrm{OH}) \cdot 2 \mathrm{SiO}_{2}\right.$, 15-20 wt.\%, $\geq 99.99 \%$, Aldrich), colloidal silica suspension (CSS) $\left(\mathrm{SiO}_{2}\right.$, LUDOX HS-30 colloidal silica, $30 \mathrm{wt} . \%$ suspension in $\mathrm{H}_{2} \mathrm{O}$, Aldrich), and tetraethylorthosilicate (TEOS) $\left(\mathrm{Si}\left(\mathrm{OC}_{2} \mathrm{H}_{5}\right)_{4}, 98 \%\right.$, Acros Organics). The organic templates added to synthesis media to aid the polymerization/organization of zeolite building blocks were tetramethylammonium hydroxide pentahydrate $\left(\left(\mathrm{CH}_{3}\right)_{4} \mathrm{~N}(\mathrm{OH}) \cdot 5 \mathrm{H}_{2} \mathrm{O}\right.$, $98 \%$, Alfa Aesar), tetra-n-propylammonium hydroxide solution $\left(\left(\mathrm{CH}_{3} \mathrm{CH}_{2} \mathrm{CH}_{2}\right)_{4} \mathrm{~N}(\mathrm{OH}), 40\right.$ wt.\% in $\mathrm{H}_{2} \mathrm{O}$, Alfa Aesar), and tetraethylammonium hydroxide $\left(\left(\mathrm{C}_{2} \mathrm{H}_{5}\right)_{4} \mathrm{~N}(\mathrm{OH}), \sim 40 \%\right.$ in $\mathrm{H}_{2} \mathrm{O}$, Aldrich). Aluminium isopropoxide $\left(\mathrm{Al}\left(\mathrm{OCH}\left(\mathrm{CH}_{3}\right)_{2}\right)_{3}\right.$, $\geq 98 \%$, Aldrich) was used as aluminium source in zeolite molecular sieves syntheses except for silicalite-1. Other reagents were phosphoric acid $\left(\mathrm{H}_{3} \mathrm{PO}_{4}, 85 \%\right.$, Junsei Chemical Co. Ltd.), sodium hydroxide ( $\mathrm{NaOH}, 97 \%$, Junsei Chemical Co. Ltd.), and hydrofluoric acid (HF, 49-52\%, Reagents Duksan). All chemicals used in the study were of analytical grade and ultrapure water $\left(0.054 \mu \mathrm{Scm}^{-1}\right)$ obtained from $\mu$ Pure system (ROMAX, Human Science, Republic of Korea) was used throughout the experiments.

Zeolite molecular sieves (MFI, FAU, LTA, and AFI) having different porous character (structure and size), surface morphology, shape, size, and most importantly Si/Al ratios have been synthesized by liquid phase routes using microwave hydrothermal reactor (CEM, Discover-909150, maximum power of $300 \mathrm{~W}$ ). The reported liquid phase microwave synthesis process consists of 3 steps: aging of precursors' reaction mixture at $25^{\circ} \mathrm{C}$ for $24 \mathrm{~h}$, crystallization at $75^{\circ} \mathrm{C}$ for $1 \mathrm{~h}$, and finally crystallization at $150^{\circ} \mathrm{C}$ for $1.5 \mathrm{~h}$ (AFI zeolite molecular sieves synthesis) with medium speed stirring. Although the aging of precursors reaction mixture was made in the microwave hydrothermal reactor, it does not make any impact on the characteristics of final product as aging in open atmosphere results in similar morphology and crystallinity product to that of microwave aging. Detailed synthesis conditions with chemical compositions and their codes have been summarized in Table 1. In all the syntheses, hydrothermal precursor gel of around $21 \mathrm{~g}$ was loaded in a $35 \mathrm{~mL}$ glass vial which was then capped with silicone/polytetrafluoroethylene and placed in microwave hydrothermal reactor. After the synthesis, the resultant milky suspensions containing nanosized zeolite molecular sieves were cooled to room temperature and diluted with deionized water. The resulting nanocrystals were then separated from mother liquor by high speed centrifugation at $15000 \mathrm{rpm}$ for $20 \mathrm{~min}$ followed by repeated redispersion in deionized water and centrifugation (washing step was repeated 5 times).

2.2. Materials and Phase Pure Nanomolecular Sieves Synthesis. Powder X-ray diffraction studies of zeolite samples were made on PANalytical X'Pert PRO diffractometer with $\mathrm{Cu}-\mathrm{K} \alpha$ radiation $(\lambda=1.5418 \AA)$ and the data was collected in $2 \theta$ range $5-60^{\circ}$ with a step size of $0.02^{\circ} \mathrm{s}^{-1}$. Phase identification was performed with the help of JCPDS files for inorganic compounds $\left(\mathrm{MFI}_{\infty} \# 98-004-1052\right.$ and \#48-0136, $\mathrm{MFI}_{250}$ and MFI $_{100} \#$ 98-006-2274, FAU \#98-015-3354, LTA \#97-002-4901, and AFI \#98-009-1674). In addition, surface morphological studies of zeolite molecular sieves particles were also made with scanning electron microscope (SEM, JEOL-JSM-7000F) and the elemental composition was measured with energy dispersive X-ray spectrometry (EDS). The particle size and particle size distributions were measured by light scattering analysis (Nanotrac Wave, Microtrac, Inc.) at $298 \mathrm{~K}$. The light provides a beam of wavelength $(\lambda)$ at $780 \mathrm{~nm}$ which is scattered by the velocities of the particles so that the signal frequency shifts according to the Doppler Effect. The power spectrum of the interference signal is calculated by a high speed Fourier transform digital signal processor and inverted to give particle size distribution (PSD). The PSD in volume percent were calculated by Microtrac FLEX 11 operating software.

\section{Results and Discussion}

Nanomolecular sieves of different $\mathrm{Si} / \mathrm{Al}$ ratio (infinite, high, moderate, low, and zero) have been successfully synthesized 
TABLE 1: Detailed reaction conditions and sample code for each as-synthesized zeolite nanomolecular sieve.

\begin{tabular}{|c|c|c|c|c|c|c|c|c|c|}
\hline \multirow{3}{*}{ Code $^{a}$} & \multirow{3}{*}{ Molar composition of reaction mixture } & \multirow{3}{*}{$\begin{array}{l}\mathrm{Si} / \mathrm{Al} \\
\text { ratio }\end{array}$} & \multirow{3}{*}{$\begin{array}{l}\text { Silica } \\
\text { source }\end{array}$} & \multicolumn{6}{|c|}{ Synthesis conditions } \\
\hline & & & & \multicolumn{2}{|c|}{ Step I } & \multicolumn{2}{|c|}{ Step II } & \multicolumn{2}{|c|}{ Step III } \\
\hline & & & & $\begin{array}{c}\text { Temp. } \\
{ }^{\circ} \mathrm{C}\end{array}$ & $\begin{array}{c}\text { Time, } \\
\mathrm{h}\end{array}$ & $\begin{array}{c}\text { Temp. } \\
{ }^{\circ} \mathrm{C}\end{array}$ & $\begin{array}{c}\text { Time, } \\
\mathrm{h}\end{array}$ & $\begin{array}{c}\text { Temp. } \\
{ }^{\circ} \mathrm{C}\end{array}$ & $\begin{array}{c}\text { Time, } \\
\mathrm{h}\end{array}$ \\
\hline $\mathrm{MFI}_{\infty}$ & $25 \mathrm{SiO}_{2}: 10 \mathrm{TPAOH}: 485 \mathrm{H}_{2} \mathrm{O}$ & $\infty$ & TEOS & 25 & 24.0 & 75 & 1.0 & 150 & 1.5 \\
\hline $\mathrm{MFI}_{250}$ & $25 \mathrm{SiO}_{2}: 0.05 \mathrm{Al}_{2} \mathrm{O}_{3}: 10 \mathrm{TPAOH}: 485 \mathrm{H}_{2} \mathrm{O}$ & 250 & TEOS & 25 & 24.0 & 75 & 1.0 & 150 & 1.5 \\
\hline $\mathrm{MFI}_{100}$ & $25 \mathrm{SiO}_{2}: 0.13 \mathrm{Al}_{2} \mathrm{O}_{3}: 10 \mathrm{TPAOH}: 485 \mathrm{H}_{2} \mathrm{O}$ & 100 & TEOS & 25 & 24.0 & 75 & 1.0 & 150 & 1.5 \\
\hline $\mathrm{FAU}_{4.4}$ & $25 \mathrm{SiO}_{2}: 2.87 \mathrm{Al}_{2} \mathrm{O}_{3}: 13.75$ (TMA) $)_{2} \mathrm{O}: 0.025 \mathrm{Na}_{2} \mathrm{O}: 1625 \mathrm{H}_{2} \mathrm{O}$ & 4.4 & CSS & 25 & 24.0 & 75 & 1.0 & 150 & 8.0 \\
\hline $\mathrm{LTA}_{1}$ & $25 \mathrm{SiO}_{2}: 12.5 \mathrm{Al}_{2} \mathrm{O}_{3}: 13.75(\mathrm{TMA})_{2} \mathrm{O}: 2 \mathrm{Na}_{2} \mathrm{O}: 1625 \mathrm{H}_{2} \mathrm{O}$ & 1 & TMAS & 25 & 24.0 & 75 & 1.0 & 150 & 1.5 \\
\hline $\mathrm{AFI}_{0}$ & $\mathrm{Al}_{2} \mathrm{O}_{3}: 1.10 \mathrm{P}_{2} \mathrm{O}_{5}: 3.5$ TEAOH $: 250 \mathrm{H}_{2} \mathrm{O}: 1.5 \mathrm{HF}$ & 0 & - & 25 & 24.0 & 75 & 1.0 & 175 & 12.0 \\
\hline
\end{tabular}

${ }^{\text {a }}$ Subscript in the sample code represents the $\mathrm{Si} / \mathrm{Al}$ ratio of reaction mixture.

using microwave hydrothermal reactor for their greater application in separation science, petrochemicals, catalytic science, sensors, and so forth. As reported in Table 1, synthesis of FAU and AFI zeolite types nanomolecular sieve took little longer synthesis time, because it has always been difficult to synthesize structure sensitive zeolite molecular sieve such as FAU zeolite type and morphology sensitive AFI zeolite type where controlled addition of alkali and acidic solution is a must condition to have desired phase and morphology, respectively; otherwise, fast structural transformation of FAU to LTA type zeolitic framework will take place. This is the main reason for a little longer crystallization ( $8 \mathrm{~h}$ FAU and $12 \mathrm{~h} \mathrm{AFI}$ ) at $150^{\circ} \mathrm{C}$ in comparison to $1.5 \mathrm{~h}$ crystallization for all other zeolite types. However, these 8 and $12 \mathrm{~h}$ of crystallization are much shorter than those of days of crystallization by conventional heating process. The following paragraphs are devoted to the detailed discussion of individual zeolite nanomolecular sieves of different $\mathrm{Si} / \mathrm{Al}$ ratios.

\subsection{Pure Silica $M F I_{\infty}$ (Silicalite-1) Zeolite Nanomolecular} Sieve. SEM micrograph of pure silica MFI zeolite type molecular sieves (Figure $1, \mathrm{MFI}_{\infty}$ ) shows the formation of well-developed nanocrystals with regular morphology. These nanocrystals have smooth edged hexagonal lump/disk-like shape of size around $200 \mathrm{~nm}$. The XRD pattern reported in Figure 2, $\mathrm{MFI}_{\infty}$, represents the high phase purity of as-synthesized silicalite-1 zeolite molecular sieves. The PSD curve of the same sample reported in Figure $3, \mathrm{MFI}_{\infty}$, reveals the narrow particle size distribution which means all the particles are of similar sizes. Silicalite- 1 has been one of the most studied materials for gas separation, catalytic reactor, pervaporation, and so forth, because of its size and shape selectivity properties. The MFI type zeolite framework structure is described as a combination of two interconnected channel systems. It consists of a system of sinusoidal channels (along $x)$ with a circular cross section $(5.1 \AA \times 5.5 \AA)$ which are interconnected with straight channels (along $y$ ) of elliptic cross section $(5.3 \AA \times 5.6 \AA)$. Both channels are defined by 10 -member rings (MR) [21]. Furthermore, the pure silica composition induces hydrophobic character, high thermal and chemical stability, and resistance to acidic environment. Due to the nonpolar framework structure of silicalite-1, it is of great demand in membrane science for the separation of mixtures of light hydrocarbons.

3.2. High Silica $M F I_{250}$ and $M F I_{100}(Z S M-5)$ Zeolite Nanomolecular Sieve. The high silica ZSM-5 zeolite is of great interest as it displays unique catalytic properties, making it useful in a number of petrochemical and oil refining processes [22]. ZSM-5 with the MFI zeolite type structure has been recognized as a prime candidate for the practical catalytic cracking because of its considerable resistance to deactivation by coking as well as its strong acidity [23]. So, we decided to synthesize ZSM-5 zeolite nanomolecular sieves with high silica content. SEM images of $\mathrm{MFI}_{250}$ and $\mathrm{MFI}_{100}$ reported in Figure 1 reveal that the ZSM-5 samples have different shape, size, and morphology. $\mathrm{MFI}_{250}$ zeolite sample contains distorted hexagonal lump/disk shape nanocrystals of size around $200 \mathrm{~nm}$, whereas the $\mathrm{MFI}_{100}$ zeolite crystals look like pseudo spheres and their sizes are much bigger than $\mathrm{MFI}_{250}$ zeolite crystals. The average size of $\mathrm{MFI}_{100}$ zeolite crystals is around $450 \mathrm{~nm}$. A close look at $\mathrm{MFI}_{100}$ zeolite crystals indicates that each nanosphere is further composed of nanocrystallites of size 5-10 nm (Figure 1, $\mathrm{MFI}_{100}$ ). Even though with the change in $\mathrm{Si} / \mathrm{Al}$ ratio a change in shape, size, and morphology of MFI zeolite nanomolecular sieves is observed (Figure 1, $\mathrm{MFI}_{250}$ and $\mathrm{MFI}_{100}$ ), framework structure remains intact (Figure 2, $\mathrm{MFI}_{250}$ and $\mathrm{MFI}_{100}$ ). The XRD patterns of both samples corresponded well with that of MFI zeolite molecular sieves with the earlier reported and JCPDS data file \#98-006-2274 [23]. These diffraction patterns also reveal the high phase purity of as-synthesized ZSM5 zeolite nanomolecular sieve samples as no other phase was observed. Although the peak positioning of diffraction patterns is the same for both samples $\left(\mathrm{MFI}_{250}\right.$ and $\left.\mathrm{MFI}_{100}\right)$, a remarkable decrease in the intensity of characteristic peak at $23.2^{\circ}$ diffraction from plane (501) is observed (represented by dotted rectangle and an arrow in Figure 2, $\mathrm{MFI}_{250}$ and $\mathrm{MFI}_{100}$ ) with shape and morphology variation. Light scattering analyses (Figure 3, $\mathrm{MFI}_{250}$ and $\mathrm{MFI}_{100}$ ) show that $\mathrm{MFI}_{250}$ zeolite molecular sieve sample consists of fine nanoparticles (narrow PSD) of size around $250 \mathrm{~nm}$, whereas crystals of $\mathrm{MFI}_{100}$ zeolite molecular sieve sample have wider PSD. The PSD results (Figure 3, $\mathrm{MFI}_{250}$ and $\mathrm{MFI}_{100}$ ) confirm 

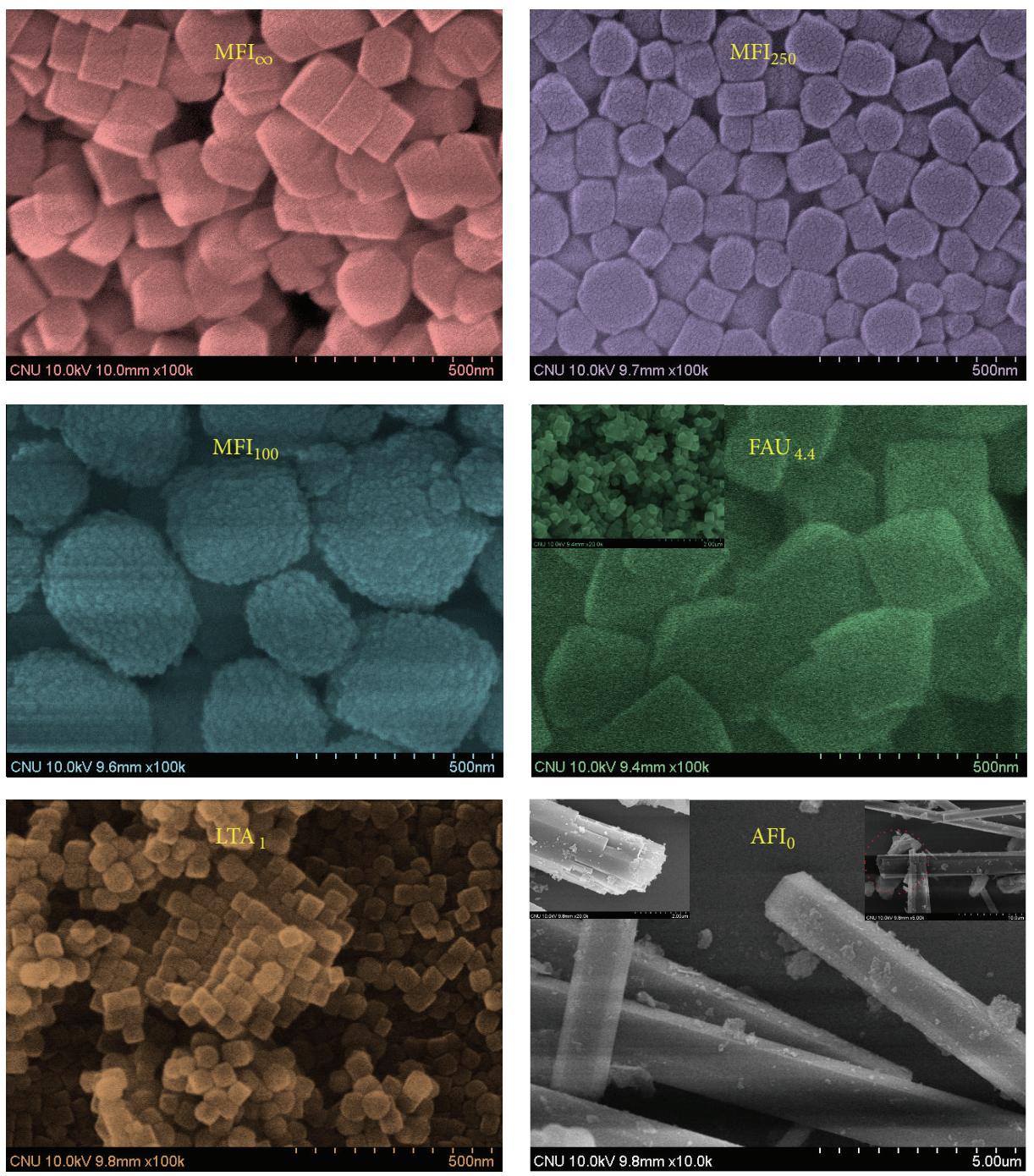

FIGURE 1: SEM images of as-synthesized zeolite nanomolecular sieves having different Si/Al ratios, particle morphologies, and sizes.

the observations made from SEM analysis (Figure 1, $\mathrm{MFI}_{250}$ and $\mathrm{MFI}_{100}$ ).

3.3. Moderate Silica $\mathrm{FAU}_{4.4}$ (NaY) Zeolite Nanomolecular Sieve. FAU-type zeolite has unique properties with larger zeolitic pore $(7.4 \AA)$, cage-like framework structure, hydrophilic character, ion-exchange property, and higher polarity so that the FAU-type zeolite materials could be multipurpose separators for dehydration, organic-organic separation such as polar/nonpolar mixtures, gas separation such as $\mathrm{CO}_{2}$ extraction, vapor-gas separation, and so forth, [24]. The FAU zeolite type (zeolite $\mathrm{Y}$ ) is also the main component of fluid catalytic cracking (FCC) catalysis. Furthermore, pervaporation process has gained widespread acceptance in the chemical industry as an effective process for separation of azeotropic mixtures and $\mathrm{NaY}$ zeolite membranes show high alcohol selectivity in the separation of alcohol-benzene, cyclohexane, or methyl tert-butyl ether $[10,24]$. Due to the versatile nature of $\mathrm{NaY}$ (FAU) zeolite, in the present work, the same has been synthesized as a potential zeolite molecular sieve with moderate silica content.

SEM images of as-synthesized FAU zeolite nanomolecular sieve by using CSS as silica source are presented in Figure 1, $\mathrm{FAU}_{4.4}$. These images show octahedron morphology of FAU zeolite nanocrystals. The particle size evaluation of this sample reveals that $\mathrm{FAU}_{4.4}$ zeolite nanomolecular sieve sample has particles of size around $150 \mathrm{~nm}$ with smooth faces and sharp edges (Figure 1, $\mathrm{FAU}_{4.4}$ ). But low magnification top-left inset image in Figure 1, $\mathrm{FAU}_{4.4}$, reveals the existence of bigger size aggregates. A close observation of the XRD pattern of $\mathrm{FAU}_{4.4}$ zeolite sample confirms the FAU zeolite phase formation with small LTA impurity phase (Figure 2, $\mathrm{FAU}_{4.4}$ ). Furthermore, the two distinct PSD curves (Figure 3, $\mathrm{FAU}_{4.4}$ ) obtained for $\mathrm{FAU}_{4.4}$ zeolite nanomolecular sieve sample confirm the existence of two kinds of particles, mainly nanosized FAU zeolite crystals (mean size $\sim 175 \mathrm{~nm}$ ) along with bigger size aggregates of size around $1500 \mathrm{~nm}$. 


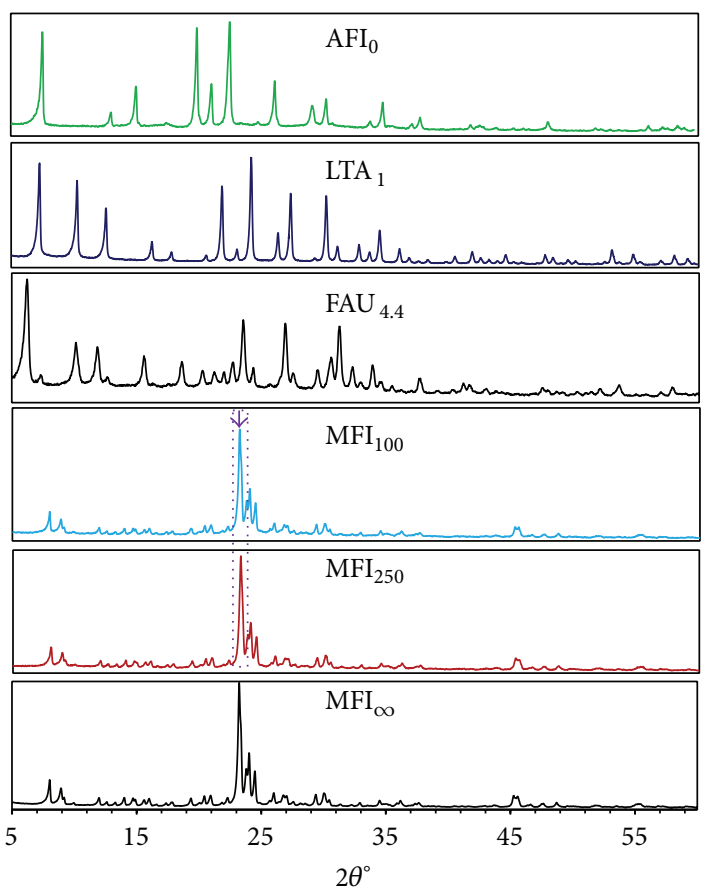

FIGURE 2: XRD patterns of microwave assisted as-synthesized zeolite nanomolecular sieves.

3.4. Low Silica LTA (NaA) Zeolite Nanomolecular Sieve. As we discussed in the previous subsections that high and moderate level silica containing zeolite molecular sieves are of paramount importance, similarly, the following couple of paragraphs will be devoted to the importance and applicability of low silica/high alumina zeolite molecular sieves in the different fields of science.

LTA zeolite is considered the first synthetic zeolite as well as highly commercialized zeolite molecular sieve. Nanostructure LTA zeolite has high hydrophilicity, maximum number of exchangeable cations, microporous character with channel opening size of $4.1 \AA$ (NaA zeolite), and so forth. The SEM image of $\mathrm{LTA}_{1}$ zeolite nanomolecular sieve sample (Figure 1, $\mathrm{LTA}_{1}$ ) demonstrates the uniform growth of LTA zeolite cubic crystals. SEM micrograph as well as LSA data (Figure 3, $\mathrm{LTA}_{1}$ ) reveals the narrow PSD of cubic crystal and the average size of each cube is around $60 \mathrm{~nm}$. Comparing the SEM micrographs (Figure 1) and PSD peak positioning reported in Figure 3, we found that $\mathrm{LTA}_{1}$ zeolite cubes are smallest in size among all other zeolite molecular sieves reported in this paper. XRD pattern (Figure 2, $\mathrm{LTA}_{1}$ ) confirms the crystal structure and high phase purity of as-synthesized LTA $_{1}$ zeolite nanomolecular sieve.

3.5. Silica-Free $\mathrm{AFI}_{0}\left(\mathrm{AlPO}_{4}-5\right)$ Nanomolecular Sieve. Traditionally, the definition of the zeolite-type materials was porous crystalline solid having three-dimensional framework structures consisting of silicon, aluminum, oxygen, and exchangeable cations. In 1982, Wilson and coworkers developed a new class of zeolite-like inorganic crystals denoted by $\mathrm{AlPO}_{4}-\mathrm{n}$ ( $\mathrm{n}$ indicates a structural type), whose structures are constructed with $\mathrm{AlO}_{4}$ and $\mathrm{PO}_{4}[25,26]$. From the past decade, $\mathrm{AlPO}_{4}$-n molecular sieves have been extensively applied in separation science, sensors, optics, and so forth. Due to the microporous character, these molecular sieves find greater utility in the separation and recovery of gases from shale gas and flue gas.

$\mathrm{AFI}_{0}$ molecular sieve crystals synthesized in dilute condition and with HF acid added in a single step exhibit rodlike morphology with dimensions of 5-50 $\mu \mathrm{m}$ in length and $1-4 \mu \mathrm{m}$ in cross section diameter (Figure $1, \mathrm{AFI}_{0}$ ). Top-left inset SEM image in Figure 1, $\mathrm{AFI}_{0}$, indicates that each micron size AFI rod is composed of a number of small sizes rods and the top-right inset image confirms our claim. The area highlighted by the dotted circle reveals oriented arrangement of crystals and a close view asserts that in actual rod crystals were composed of flat sticks shape crystals (top-right inset, Figure $1, \mathrm{AFI}_{0}$ ). The broad diffraction peaks that appeared in the XRD pattern of $\mathrm{AFI}_{0}$ molecular sieve (Figure $3, \mathrm{AFI}_{0}$ ) may correspond to these ultrathin films (sticks). The PSD curve reveals the broad size distribution of $\mathrm{AFI}_{0}$ molecular sieves and crystals size lies in micron scale.

In order to achieve the nano-AFI $\left(\mathrm{AlPO}_{4}-5\right)$ molecular sieve, a number of experiments were performed by controlled addition of HF acid, crystallization time, aging time, and so forth. The SEM micrographs of as-synthesized ultrathin $\mathrm{AlPO}_{4}-5$ molecular sieves obtained from the same chemical composition and by controlled $\mathrm{HF}$ acid addition ( $48 \mathrm{~h}$ aging, two-step crystallization $75^{\circ} \mathrm{C}(2 \mathrm{~h}), 165^{\circ} \mathrm{C}(3 \mathrm{~h}$ crystallization $\mathrm{AlPO}_{4}-5^{3 \mathrm{~h}}$ (sample 1), $10 \mathrm{~h}$ crystallization $\mathrm{AlPO}_{4}-5^{10 \mathrm{~h}}$ (sample 2))) have been shown in Figure 4. The images reveal the successful synthesis of flat stick shape $\mathrm{AlPO}_{4}-5$ nanocrystal with some impurity phase. The preliminary information 

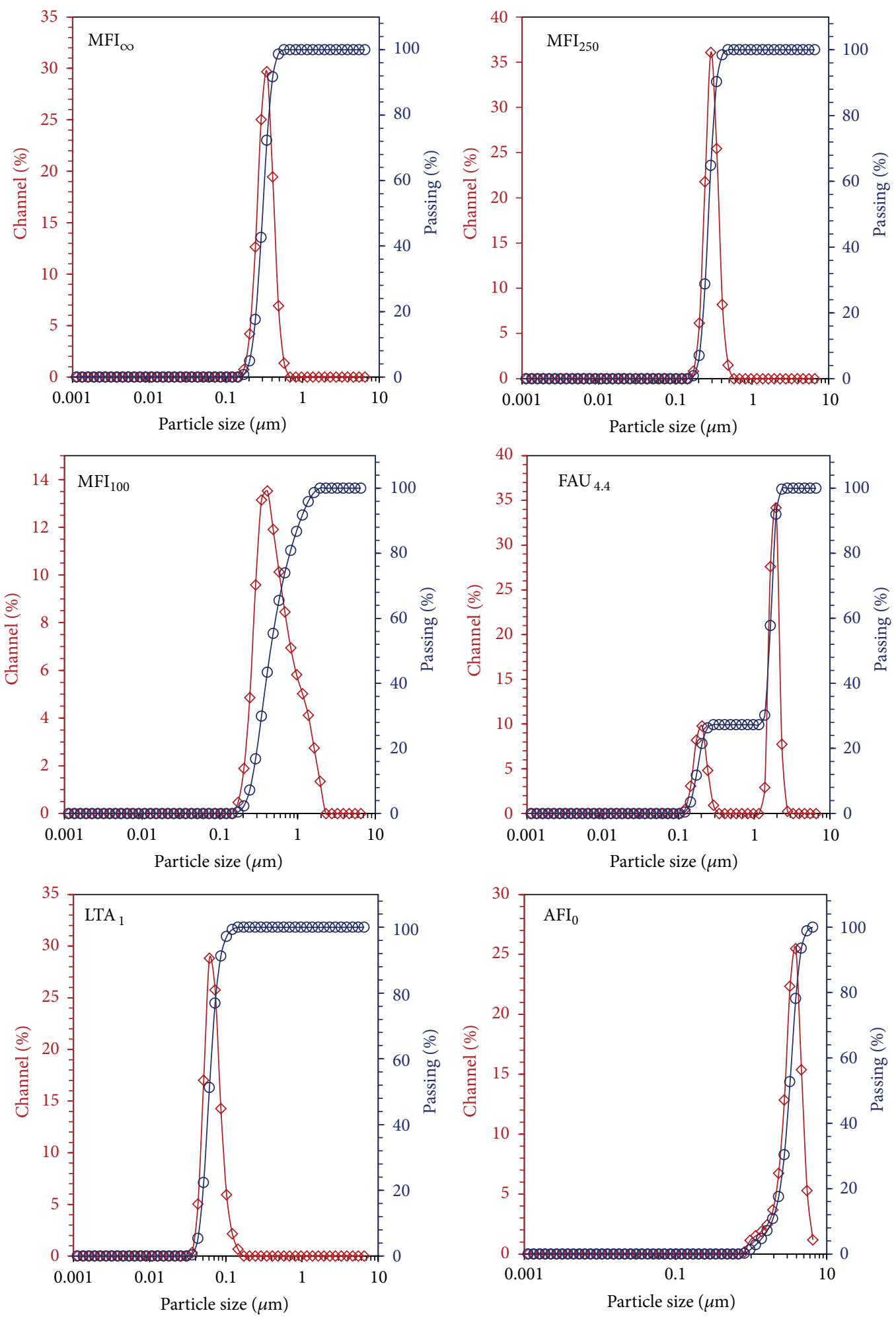

FIGURE 3: Particle size distribution curves of microwave assisted as-synthesized zeolite nanomolecular sieves.

suggests that by controlling the synthesis condition and initial composition of reaction mixture we can synthesize uniform size ultrathin $\mathrm{AlPO}_{4}-5$ nanomolecular sieve which has not been reported earlier.
3.6. Elemental Analysis. Quantitative elemental analyses of as-synthesized zeolite nanomolecular sieves have been made by selected area electron dispersive spectroscopy. The outcomes of EDS studies have been reported in the form of EDS 



Figure 4: Two different magnification SEM images of $\mathrm{AlPO}_{4}-5$ molecular sieves synthesized at $165^{\circ} \mathrm{C}$ temperature under different crystallization times: $\mathrm{AlPO}_{4}-5^{3 \mathrm{~h}}(3 \mathrm{~h})$ and $\mathrm{AlPO}_{4}-5^{10 \mathrm{~h}}(10 \mathrm{~h})$.

spectra in Figure 5, and the relative proportions of elements in terms of weight $\%$ and atomic $\%$ are reported in Table 2. EDS spectra of MFI type zeolite molecular sieves show strong elemental signal only for oxygen and silicon, as the aluminum content is very, very low in these samples (pure silica $\mathrm{MFI}_{\infty}$ zeolite sample). On the other hand, the intensity of aluminum signal increases for aluminum rich zeolite molecular sieves in the following order: $\mathrm{FAU}_{4.4}<\mathrm{LTA}_{1}<\mathrm{AFI}_{0}$ (Figure 5). Atomic $\%$ data tabulated in Table 2 reveals that the elemental molar ratio of the final product is quite close to the composition of the starting materials and desired $\mathrm{Si} / \mathrm{Al}$ ratios.

\section{Conclusion}

Nanosized zeolite molecular sieves of different Si/Al ratio ranging from infinity to zero, with independent shape, size, morphology, crystal structure, microporosity, hydrophilicity, and so forth, have been successfully synthesized using MHR. These nanomolecular sieves shall be utilized in hybrid membrane synthesis, zeolite membrane seeding, catalytic application, and gas adsorption studies in our future course of work. Ultrathin flat stick shape AFI nanocrystals with high aspect ratio will be more useful for the fabrication of mixed matrix membrane if we could horizontally align them on/in polymeric membrane surface. The most important characteristic of these lamellar materials (sheet shape inorganic filler of aspect ratio $>10$ ) is that, by carefully manipulating the orientation of their packing order, an ultrathin skin layer can be obtained with no sacrifice of selectivity compared to conventional filler with low aspect ratio morphology [27]. Although we did not perform growth mechanism studies for
TABLE 2: EDS analysis results of microwave assisted as-synthesized zeolite nanomolecular sieves.

\begin{tabular}{|c|c|c|c|c|c|}
\hline \multirow{2}{*}{ Sample } & \multirow{2}{*}{$\mathrm{Si}: \mathrm{Al}^{\mathrm{a}}$} & \multicolumn{4}{|c|}{ EDS analysis results } \\
\hline & & Element & Weight \% & Atomic \% & Atomic $\mathrm{Si}: \mathrm{Al}$ \\
\hline \multirow{2}{*}{$\mathrm{MFI}_{\infty}$} & \multirow{2}{*}{$\infty$} & $\mathrm{OK}$ & 54.25 & 67.55 & \multirow{2}{*}{$\infty$} \\
\hline & & Si K & 45.75 & 32.45 & \\
\hline \multirow{3}{*}{$\mathrm{MFI}_{250}$} & \multirow{3}{*}{250} & $\mathrm{OK}$ & 49.10 & 62.87 & \multirow{3}{*}{246.5} \\
\hline & & $\mathrm{Al} \mathrm{K}$ & 0.20 & 0.15 & \\
\hline & & Si K & 50.70 & 36.98 & \\
\hline \multirow{3}{*}{$\mathrm{MFI}_{100}$} & \multirow{3}{*}{100} & $\mathrm{OK}$ & 51.53 & 65.10 & \multirow{3}{*}{98.7} \\
\hline & & $\mathrm{Al} \mathrm{K}$ & 0.47 & 0.35 & \\
\hline & & Si K & 48.00 & 34.55 & \\
\hline \multirow{4}{*}{ FAU } & \multirow{4}{*}{4.4} & $\mathrm{OK}$ & 50.77 & 63.37 & \multirow{4}{*}{2.08} \\
\hline & & $\mathrm{Na} \mathrm{K}$ & 7.90 & 6.87 & \\
\hline & & $\mathrm{Al} \mathrm{K}$ & 13.07 & 9.67 & \\
\hline & & Si K & 28.26 & 20.09 & \\
\hline \multirow{4}{*}{ LTA } & \multirow{4}{*}{1} & $\mathrm{OK}$ & 49.65 & 61.95 & \multirow{4}{*}{1.30} \\
\hline & & $\mathrm{Na} \mathrm{K}$ & 11.39 & 9.89 & \\
\hline & & $\mathrm{Al} \mathrm{K}$ & 16.56 & 12.25 & \\
\hline & & Si K & 22.40 & 15.92 & \\
\hline \multirow{3}{*}{ AFI } & \multirow{3}{*}{0} & $\mathrm{OK}$ & 58.46 & 71.79 & \multirow{3}{*}{0.00} \\
\hline & & $\mathrm{Al} \mathrm{k}$ & 19.74 & 14.38 & \\
\hline & & P K & 21.80 & 13.83 & \\
\hline
\end{tabular}

${ }^{\mathrm{a}}$ Molar ratio of initial reaction mixture.

any of these molecular sieves, as per reports and our earlier studies, the clear solution synthesis mainly follows growth by monomer (solution-mediated transport), and heterogeneous 

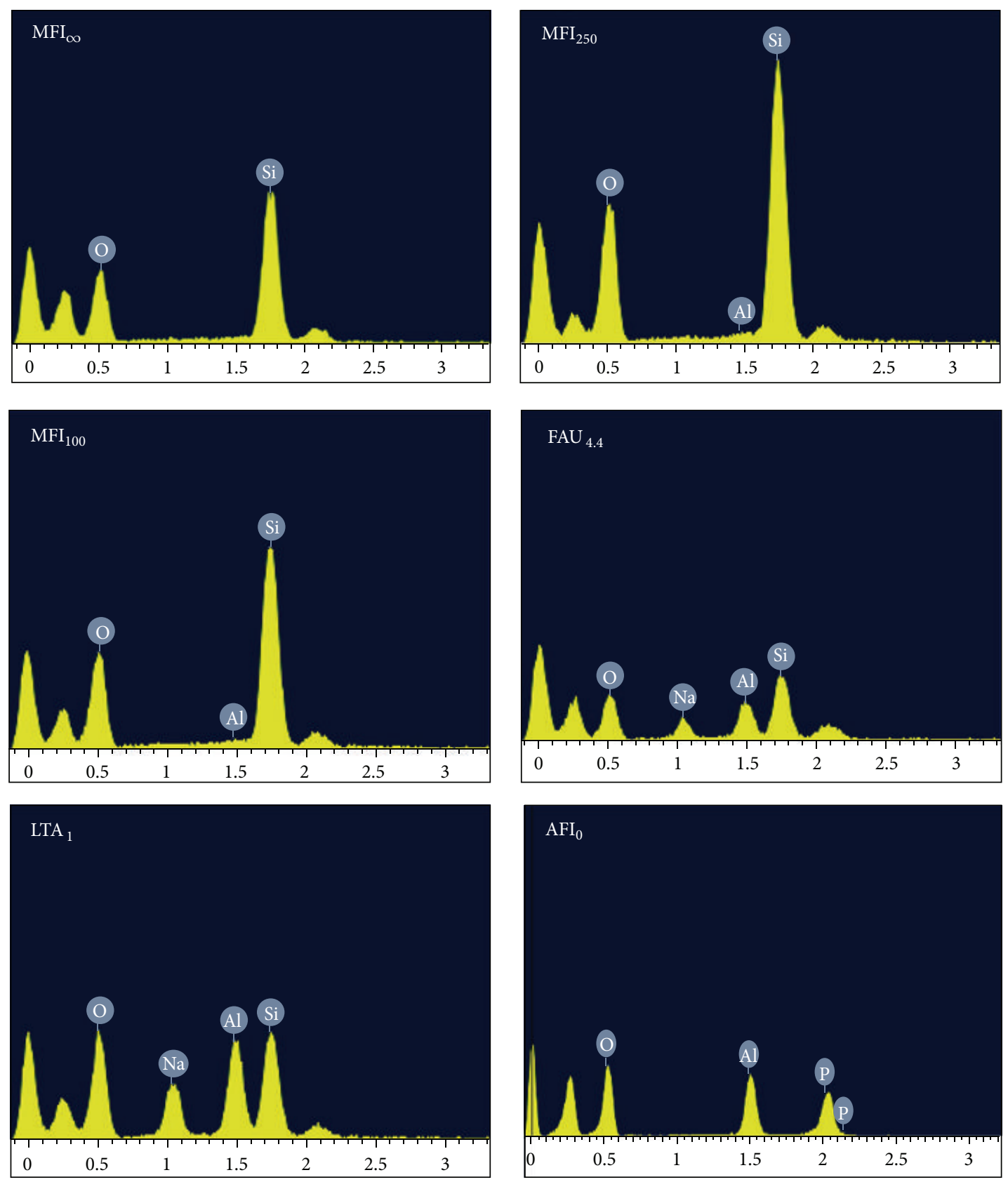

FIGURE 5: Quantitative elemental analysis spectra of microwave assisted as-synthesized zeolite nanomolecular sieves by EDS.

precipitation (use of inorganic precursors) synthesis follows aggregative growth mechanism [28, 29].

\section{Conflict of Interests}

The authors declare that there is no conflict of interests regarding the publication of this paper.

\section{Acknowledgment}

This work was supported by the Korean CCS R\&D Center (KCRC) grant (2014M1A8A1049310) funded by the Korean Government (Ministry of Science, ICT \& Future Planning).

\section{References}

[1] Y. Hu, C. Liu, Y. Zhang, N. Ren, and Y. Tang, "Microwaveassisted hydrothermal synthesis of nanozeolites with controllable size," Microporous and Mesoporous Materials, vol. 119, no. 1-3, pp. 306-314, 2009.

[2] Z. Luan, M. Hartmann, D. Zhao, W. Zhou, and L. Kevan, "Alumination and ion exchange of mesoporous SBA-15 molecular sieves," Chemistry of Materials, vol. 11, no. 6, pp. 1621-1627, 1999.

[3] A. Corma, V. Fornes, M. T. Navarro, and J. Perezpariente, "Acidity and Stability of MCM-41 Crystalline Aluminosilicates," Journal of Catalysis, vol. 148, no. 2, pp. 569-574, 1994.

[4] V. Luca, D. J. MacLachlan, R. Bramley, and K. Morgan, "Electron paramagnetic resonance and electron spin echo modulation 
study of surface sites of the porous aluminosilicate MCM41 using transition metal ion probes," The Journal of Physical Chemistry, vol. 100, no. 5, pp. 1793-1800, 1996.

[5] S. S. A. Talesh, S. Fatemi, S. J. Hashemi, and M. Ghasemi, "Effect of $\mathrm{Si} / \mathrm{Al}$ ratio on $\mathrm{CO}_{2}-\mathrm{CH}_{4}$ adsorption and selectivity in synthesized SAPO-34," Separation Science and Technology, vol. 45, no. 9, pp. 1295-1301, 2010.

[6] E. J. García, J. Pérez-Pellitero, G. D. Pirngruber et al., “Tuning the adsorption properties of zeolites as adsorbents for $\mathrm{CO}_{2}$ separation: best compromise between the working capacity and selectivity," Industrial and Engineering Chemistry Research, vol. 53, no. 23, pp. 9860-9874, 2014.

[7] J. F. Denayer, W. Souverijns, P. A. Jacobs, J. A. Martens, and G. V. Baron, "High-temperature low-pressure adsorption of branched $\mathrm{C}_{5}-\mathrm{C}_{8}$ alkanes on zeolite beta, ZSM-5, ZSM-22, zeolite Y, and mordenite," The Journal of Physical Chemistry B, vol. 102, no. 23, pp. 4588-4597, 1998.

[8] Y. Morigami, M. Kondo, J. Abe, H. Kita, and K. Okamoto, "The first large-scale pervaporation plant using tubular-type module with zeolite NaA membrane," Separation and Purification Technology, vol. 25, no. 1-3, pp. 251-260, 2001.

[9] Y. Yue, H. Liu, P. Yuan, C. Yu, and X. Bao, "One-pot synthesis of hierarchical FeZSM-5 zeolites from natural aluminosilicates for selective catalytic reduction of $\mathrm{NO}$ by $\mathrm{NH}_{3}$," Scientific Reports, vol. 5, article 9270, 2015.

[10] H. Kita, T. Inoue, H. Asamura, K. Tanaka, and K. Okamoto, "NaY zeolite membrane for the pervaporation separation ofmethanol-methyl tert-butyl ether mixtures," Chemical Communications, no. 1, pp. 45-46, 1997.

[11] X. Zou, P. Bazin, F. Zhang, G. Zhu, V. Valtchev, and S. Mintova, "Ethanol recovery from water using silicalite-1 membrane: an operando infrared spectroscopic study," ChemPlusChem, vol. 77, no. 6, pp. 437-444, 2012.

[12] C. H. Cho, K. Y. Oh, J. G. Yeo, S. K. Kim, and Y. M. Lee, "Synthesis, ethanol dehydration and thermal stability of $\mathrm{NaA}$ zeolite/alumina composite membranes with narrow nonzeolitic pores and thin intermediate layer," Journal of Membrane Science, vol. 364, no. 1-2, pp. 138-148, 2010.

[13] C. Ji, Y. Tian, Y. Li, and Y. S. Lin, "Thin oriented AFI zeolite membranes for molecular sieving separation," Microporous and Mesoporous Materials, vol. 186, pp. 80-83, 2014.

[14] J. Hua and Y. Han, "One-step preparation of zeolite silicalite1 microspheres with adjustable macroporosity," Chemistry of Materials, vol. 21, no. 12, pp. 2344-2348, 2009.

[15] N. Viswanadham, R. Kamble, M. Singh, M. Kumar, and G. M. Dhar, "Catalytic properties of nano-sized ZSM-5 aggregates," Catalysis Today, vol. 141, no. 1-2, pp. 182-186, 2009.

[16] S. Elzey, A. Mubayi, S. C. Larsen, and V. H. Grassian, "FTIR study of the selective catalytic reduction of $\mathrm{NO}_{2}$ with ammonia on nanocrystalline NaY and CuY," Journal of Molecular Catalysis A: Chemical, vol. 285, no. 1-2, pp. 48-57, 2008.

[17] Z. Lai, G. Bonilla, I. Diaz et al., "Microstructural optimization of a zeolite membrane for organic vapor separation," Science, vol. 300, no. 5618, pp. 456-460, 2003.

[18] I. Bilecka and M. Niederberger, "Microwave chemistry for inorganic nanomaterials synthesis," Nanoscale, vol. 2, no. 8, pp. 1358-1374, 2010.

[19] S. H. Jhung, J.-S. Chang, Y. K. Hwang, and S.-E. Park, "Crystal morphology control of AFI type molecular sieves with microwave irradiation," Journal of Materials Chemistry, vol. 14, no. 2, pp. 280-285, 2004.
[20] J. Motuzas, A. Julbe, R. D. Noble, C. Guizard, Z. J. Beresnevicius, and D. Cot, "Rapid synthesis of silicalite-1 seeds by microwave assisted hydrothermal treatment," Microporous and Mesoporous Materials, vol. 80, no. 1-3, pp. 73-83, 2005.

[21] R. Krishna and D. Paschek, "Molecular simulations of adsorption and siting of light alkanes in silicalite-1," Physical Chemistry Chemical Physics, vol. 3, no. 3, pp. 453-462, 2001.

[22] A. E. Persson, B. J. Schoeman, J. Sterte, and J.-E. Otterstedt, "Synthesis of stable suspensions of discrete colloidal zeolite (Na, TPA)ZSM-5 crystals," Zeolites, vol. 15, no. 7, pp. 611-619, 1995.

[23] H. Mochizuki, T. Yokoi, H. Imai et al., "Facile control of crystallite size of ZSM-5 catalyst for cracking of hexane," Microporous and Mesoporous Materials, vol. 145, no. 1-3, pp. 165-171, 2011.

[24] K. Sato, K. Sugimoto, and T. Nakane, "Synthesis of industrial scale $\mathrm{NaY}$ zeolite membranes and ethanol permeating performance in pervaporation and vapor permeation up to $130^{\circ} \mathrm{C}$ and $570 \mathrm{kPa}$, Journal of Membrane Science, vol. 310, no. 1-2, pp. 161173, 2008.

[25] M. Choi, R. Srivastava, and R. Ryoo, "Organosilane surfactantdirected synthesis of mesoporous aluminophosphates constructed with crystalline microporous frameworks," Chemical Communications, no. 42, pp. 4380-4382, 2006.

[26] S. T. Wilson, B. M. Lok, C. A. Messina, T. R. Cannan, and E. M. Flanigen, "Aluminophosphate molecular sieves: a new class of microporous crystalline inorganic solids," Journal of the American Chemical Society, vol. 104, no. 4, pp. 1146-1147, 1982.

[27] G. Dong, H. Li, and V. Chen, "Challenges and opportunities for mixed-matrix membranes for gas separation," Journal of Materials Chemistry A, vol. 1, no. 15, pp. 4610-4630, 2013.

[28] S. Mintova, N. H. Olson, V. Valtchev, and T. Bein, "Mechanism of zeolite a nanocrystal growth from colloids at room temperature," Science, vol. 283, no. 5404, pp. 958-960, 1999.

[29] P. Sharma, J.-G. Yeo, M. H. Han, and C. H. Cho, "NaA zeolite cubic crystal formation and deformation: cubes with crystalline core, simultaneous growth of surface and core crystals, and layer-by-layer destruction," RSC Advances, vol. 2, no. 20, pp. 7809-7823, 2012. 

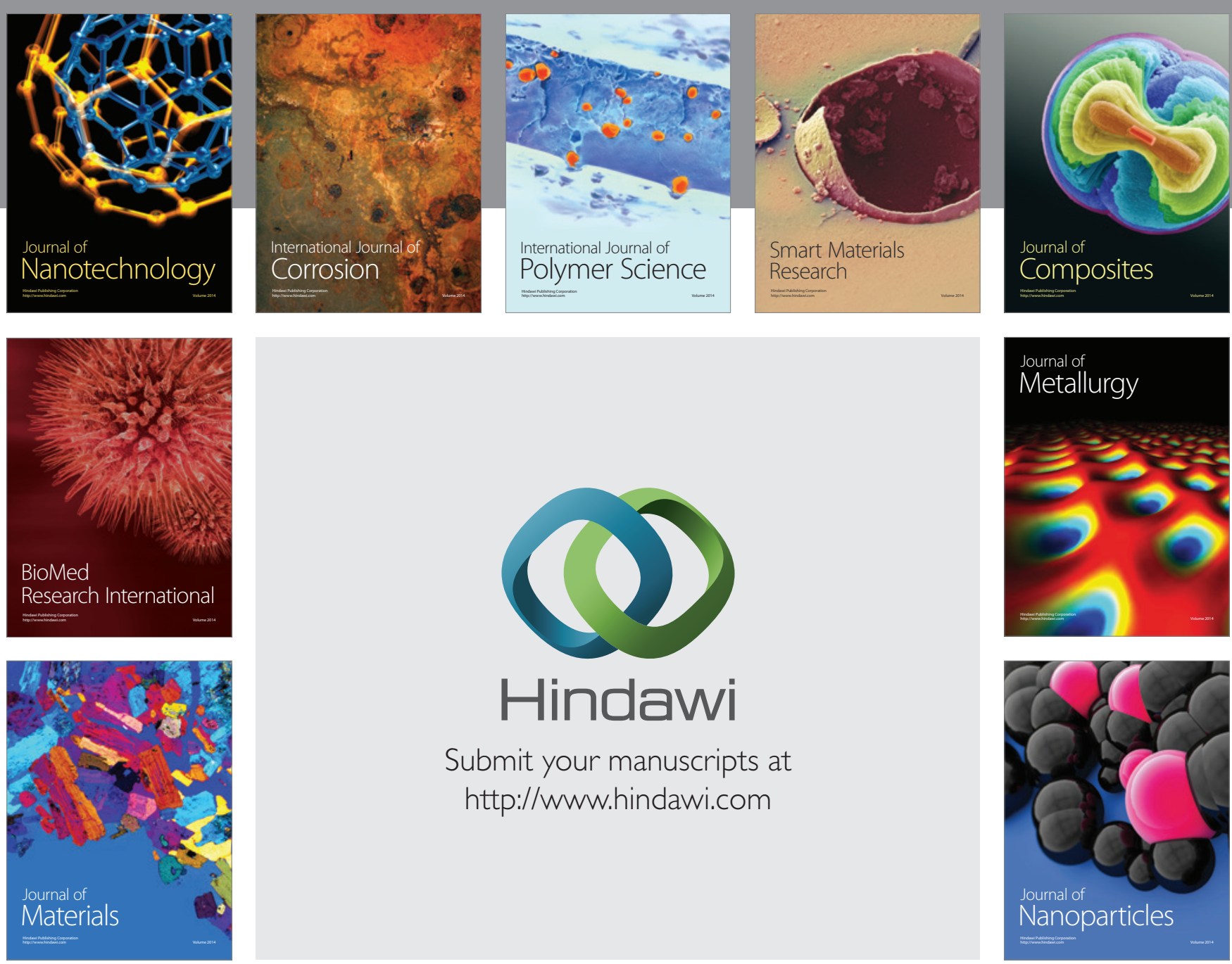

Submit your manuscripts at http://www.hindawi.com
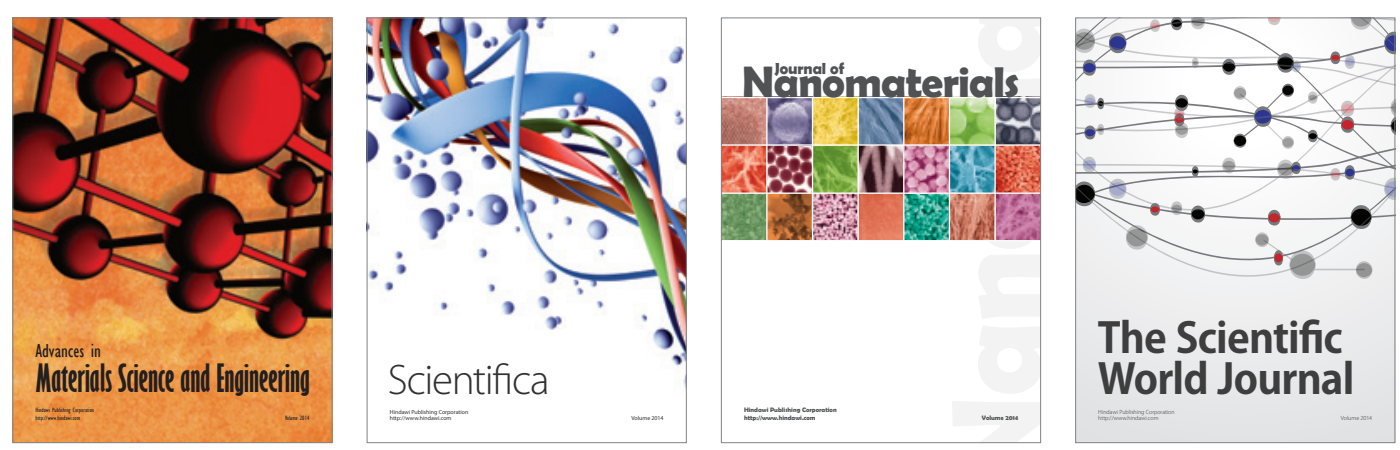

\section{The Scientific World Journal}
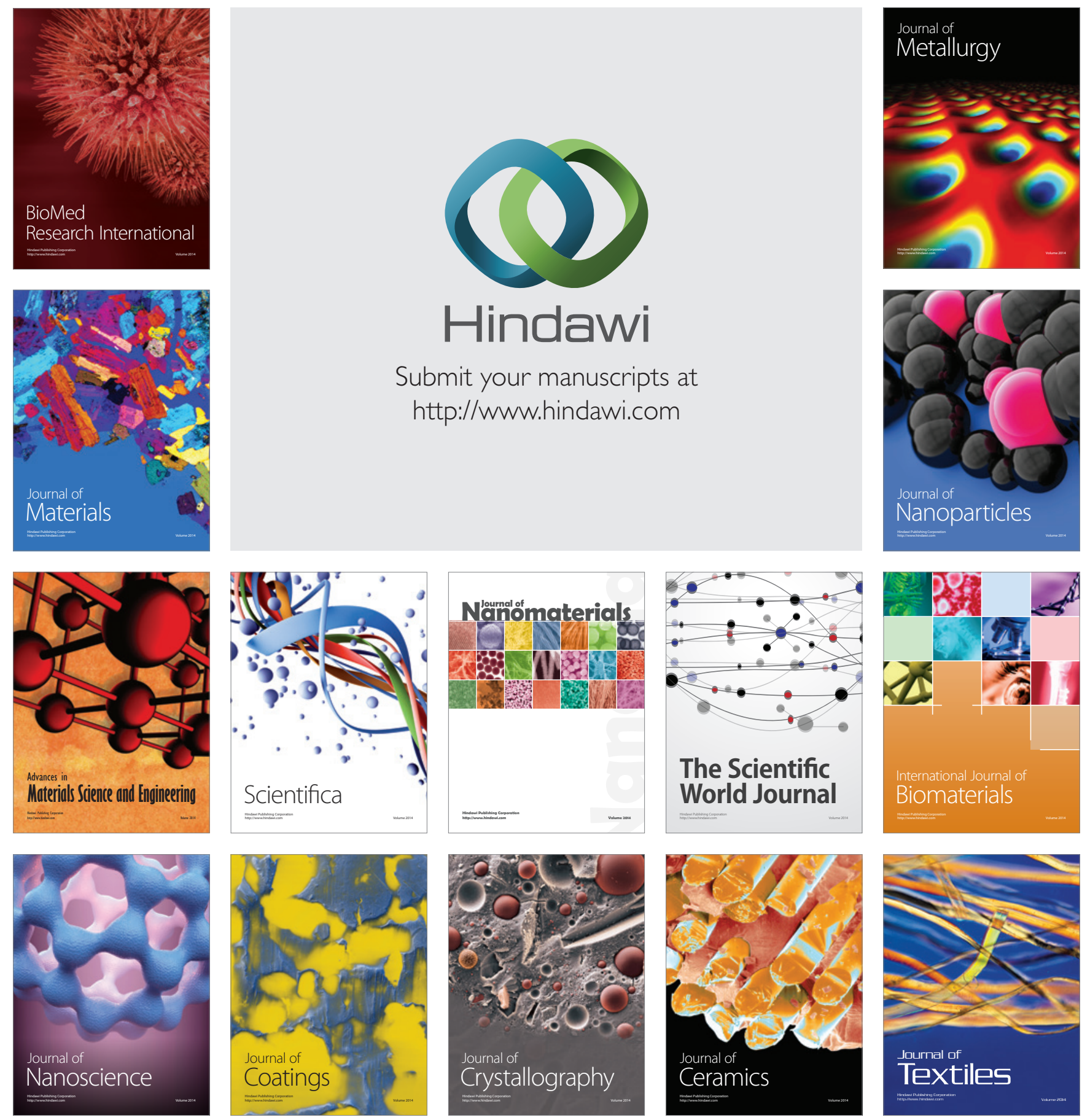